\title{
ОТДАЛЕННАЯ ПОСЛЕОПЕРАЦИОННАЯ КОГНИТИВНАЯ ДИСФУНКЦИЯ
}

\author{
${ }^{1}$ Дубовская С. С. \\ ${ }^{2}$ Товажннянская Е. Л. \\ ${ }^{3}$ Григоров Ю. Б. \\ ${ }^{4}$ Кудинова О. В. \\ ${ }^{5}$ Соловйова Е. T.
}

${ }^{1}$ к. мед. н., дочент, доиент кафедры медииины неотложных состояний, анестезиологии и интенсивной терапии

${ }^{2}$ д. мед. н., профессор, заведующая кафедры неврологии №2;

${ }^{3}$ д. мед. н., профессор, профессор кафедры хирургии № 1;

${ }^{4}$ к. мед. н., дочент, доцент кафедры медицины неотложных состояний, анестезиологии и интенсивной терапии;

${ }^{5}$ к. мед. н., ассистент кафедры неврологии №2

Украина, город Харьков, Харьковского национального медицинского университета

DOI: https://doi.org/ 10.31435/rsglobal_ws/30092018/6134

\section{ARTICLE INFO}

Received: 17 July 2018

Accepted: 21 September 2018

Published: 30 September 2018

\section{KEYWORDS}

anesthesia,

cognitive function,

neurology,

general anesthesia.

\begin{abstract}
Postoperative cognitive dysfunction develops in the early and persists in the late postoperative period. Clinically, this condition manifests itself in the form of memory impairment, violation of concentration and violation of other higher functions of the cerebral cortex. The aim of this study is to study cognitive function in patients who underwent surgery with the use of general anesthesia in a remote period in a month.

According to the results of the MMSE test, in the middle-aged patients the dynamics of the deterioration of the cognitive function was observed to a lesser degree than in the young patients, which is probably related to the age-related features of the plasticity of the cognitive function. In patients of middle age, according to the results of the FAB scale, at this period of the study was at the same level as those in young patients, which is associated with the age specificity of the plasticity of the cognitive function. According to the test drawing hours, there was no significant difference in the recovery rate, it was gradual, but no complete restoration was observed. For the 10-word memory test and the Schulte trial, the recovery was gradual, with a full recovery of the indicator to the level before the operation.
\end{abstract}

Citation: Дубовская С. С., Товажнянская Е. Л., Григоров Ю. Б., Кудинова О. В., Соловйова Е. Т. (2018) Otdalennaya Posleoperacionnaya Kognitivnaya Disfunkciya. World Science. 9(37). doi: 10.31435/rsglobal_ws/30092018/6134

Copyright: (C 2018 Дубовская С. С., Товажнянская Е. Л., Григоров Ю. Б., Кудинова О. В., Соловйова Е. Т. This is an open-access article distributed under the terms of the Creative Commons Attribution License (CC BY). The use, distribution or reproduction in other forums is permitted, provided the original author(s) or licensor are credited and that the original publication in this journal is cited, in accordance with accepted academic practice. No use, distribution or reproduction is permitted which does not comply with these terms.

Послеоперационная когнитивная дисфункция развивается в раннем и сохраняется в позднем послеоперационном периоде. Клинически данное состояние проявляется в виде нарушений памяти, нарушении концентрации внимания и нарушении других высших функций коры головного мозга [1,3].

Литературно отмечено, что возможно возникновение нарушений центральной нервной системы в послеоперационном периоде в виде: психопатологических и психотических реакций, 
делирия, судорожного синдрома, послеоперационной когнитивной дисфункции, нарушения цикла сна и бодрствования, нарушение координации, возникновение острого нарушения мозгового кровообращения, острой сенсоневральной тугоухости, спастической параплегии и прочее. Степень и выраженность патологических изменений со стороны центральной нервной системы зависит от типа анестезии, соматического состояния и неврологического статуса пациента на предоперационный период, возраста пациента и других факторов. В подавляющем большинстве случаев на фоне общего угнетения функций центральной нервной системы, наблюдается в разной степени снижение памяти, внимания, реактивности и возникают нарушения функции координации. Среди этих нарушений когнитивные изменения, возможно, сразу выделить в ходе исследования ментального статуса, характеризующееся общим видом и поведением, ориентацией, вниманием и концентрацией, эмоциональным состоянием, мышлением и познавательными процессами (память, возможность до логического суждения, речь, восприятие, праксис и исполнительные функции) [5-7].

Вообще когнитивная дисфункция связана с интегративной деятельностью головного мозга. Наиболее тонкие и важные функции и главного, которые помогают выполнять рациональное осознание мира, понимать как когнитивные функции [2,3].

Имеет большое значение в рассмотрении данной темы учитывать главные теории влияния наркоза [8].

В патогенезе возникновения когнитивной дисфункции, в послеоперационном периоде, выделяются главные факторы общей анестезии: метаболические, гемореологические, гипоксические, токсичны. Поэтому изучение этиопатогенетических механизмов когнитивной дисфункции, возникающие как следствие общей анестезии, в дальнейшем поможет разработать адекватные методы профилактики данной патологии и является актуальной задачей современной анестезиологии и неврологии.

Целью данного исследования является исследование когнитивной функции у пациентов, которым проводилось оперативное вмешательство с использованием общей анестезии в отдаленном периоде, через месяц.

Исследование было проведено в хирургических отделениях различного профиля на базе Харьковской городской клинической больницы скорой и неотложной медицинской помощи им. проф. А.И. Мещанинова. Оперативное вмешательство проводили в условиях общей многокомпонентной анестезии с искусственной вентиляцией легких с использованием пропофола и фентанила, тиопентала -натрия и фентанила.

Для достижения данной цели нами было проведено исследование когнитивной сферы у пациентов разных возрастных групп: молодого возраста, среднего возраста, пожилые люди с острой хирургической патологией до операции и на 30 сутки после оперативного вмешательства по сравнению с данными предоперационного периода.

Методы исследования. Стандартные клинические и лабораторные. Исследование когнитивной сферы: шкала MMSE, тест рисования часов, тест «10 слов», батарея тестов на лобную дисфункцию, метод Шульте. Пациенты были ознакомлены с данными о своем заболевании, объем оперативного вмешательства, возможными осложнениями. Вычисляли показатель общего когнитивного дефицита.

Динамику изменения состояния когнитивной функции у пациентов после операции с использованием общей анестезии исследованы у 130 пациентов со стандартной схемой ведения послеоперационного периода.

Проводилось нейропсихологическое тестирование по шкале MMSE, шкале FAB, теста рисования часах, теста 10 слов А. Лурия, пробы Шульте, показателя ЗКД.

Пациенты были распределены соответственно на три группы:

1 группа $(\mathrm{n}=46)$ - пациенты молодого возраста (18-44 года); средний возраст $30,1 \pm 1,0$ года, 24 человека, 22 женщины.

2 группа ( $\mathrm{n}=43)$ - пациенты среднего возраста (44-60 года); средний возраст 49,3 $\pm 5,1$ года, 18 мужчины, 25 женщины.

3 группа (n = 41) - пациенты пожилого возраста (60-80 года); средний возраст 74,4 $\pm 6,1$ года, 22 человека, 19 женщины.

Динамика результатов теста по шкале MMSE на этапах исследования.

Через месяц у пациентов всех групп после операции показатель теста по шкале MMSE был на 12,3\% ниже максимально возможного значения данного теста и на 2,7\% ниже значения по данному тесту в период до операции. Показатель теста по шкале MMSE также отличался во всех группах пациентов через 1 месяц. Через месяц исследования состояние когнитивной 
функции у пациентов значительно улучшилось, у пациентов 2 группы восстановился полностью, у пациентов 1 группы стал почти как к состоянию до операции. У пациентов 1 группы через месяц после операции показатель теста по шкале MMSE был на 6,0\% ниже максимально возможного значения по данному тесту и на 1,74\% ниже значения по данному тесту в период до операции у пациентов этой группы. У пациентов 2 группы через месяц после операции показатель теста по шкале MMSE был на 10,0\% ниже максимально возможного значения по данному тесту и на уровне значения до операции у пациентов этой группы. У пациентов 3 группы через месяц после операции показатель теста по шкале MMSE был на 20,6\% ниже максимально возможного значения по данному тесту и на 5,9\% ниже значения по данному тесту в период до операции у пациентов этой группы.

У пациентов среднего возраста наблюдалась динамика ухудшения состояния когнитивной функции в меньшей степени, чем у пациентов молодого возраста, что, возможно связано с возрастными особенностями пластичности когнитивной функции [9].

Динамика результатов шкалы FАВ на этапах исследования.

Через месяц у пациентов всех групп после операции показатель теста по шкале FAB был на 9,8\% ниже максимально возможного значения данного теста и на 2,4\% ниже значения по данному тесту в период до операции. Показатель теста по шкале FAB также отличался во всех группах пациентов через 1 месяц. Через месяц исследования состояние когнитивной функции у пациентов с тестами по шкале FAB значительно достоверно улучшилось, но полностью не восстановился у пациентов всех групп. У пациентов 1 группы через месяц после операции показатель теста по шкале FAB был на 4,4\% ниже максимально возможного значения по данному тесту и на $1,7 \%$ ниже значения по данному тесту в период до операции у пациентов этой группы. У пациентов 2 группы через месяц после операции показатель теста по шкале FAB был на 5,0\% ниже максимально возможного значения по данному тесту и на $1,1 \%$ ниже значения по данному тесту в период до операции у пациентов этой группы. У пациентов 3 группы через месяц после операции показатель теста по шкале FAB был на 20,0\% ниже максимально возможного значения по данному тесту и на 4,6\% ниже значения по данному тесту в период до операции у пациентов этой группы. У пациентов среднего возраста наблюдалась динамика восстановления состояния когнитивной функции быстрее в отношении с показателями у пациентов молодого возраста, что, возможно связано с возрастными особенностями пластичности когнитивной функции [9].

Динамика результатов теста рисования часов на этапах исследования.

Через месяц у пациентов всех групп после операции показатель теста рисования часов был на 7,7\% ниже максимально возможного значения данного теста и на 3,8\% ниже значения по данному тесту в период до операции. Показатель теста рисования часов также отличался во всех группах пациентов через 1 месяц. Через месяц исследования состояние когнитивной функции у пациентов с тестами рисования часов достоверно улучшилось, но полностью не восстановился у пациентов всех групп. У пациентов 1 группы через месяц после операции показатель теста рисования часов был на 6,0\% ниже максимально возможного значения по данному тесту и на 4,1\% ниже значения по данному тесту в период до операции у пациентов этой группы. У пациентов 2 группы через месяц после операции показатель теста рисования часов был на 7,0\% ниже максимально возможного значения по данному тесту и на 5,1\% ниже значения по данному тесту в период до операции у пациентов этой группы. У пациентов 3 группы через месяц после операции показатель теста рисования часов был 9,0 $\pm 2,7$, что на $10,0 \%$ ниже максимально возможного значения по данному тесту и на 2,1\% ниже значения по данному тесту в период до операции у пациентов этой группы. У пациентов всех групп во все сроки наблюдений существенной разницы скорости восстановления не было, оно было постепенным, но полного восстановления не наблюдалось.

Динамика результатов теста 10 слов А. Лурия на этапах исследования.

Показатель теста 10 слов также отличался во всех группах пациентов через 1 месяц. Через месяц исследования состояние когнитивной функции у пациентов с тестами 10 слов достоверно улучшилось, но полностью восстановился через месяц у пациентов 1 группы. У пациентов 1 и 3 групп через три месяца после операции показатель теста 10 слов полностью восстановился по сравнению со значениями данного теста к операции у пациентов этой группы. У пациентов 2 группы через три месяца после операции показатель теста 10 слов был на 21,0\% ниже максимально возможного значения по данному тесту и на 2,4\% ниже значения по данному тесту в период до операции у пациентов этой группы. У пациентов всех групп в короткие сроки наблюдений существенной разницы скорости восстановления не было. 
Восстановление было постепенным, с полным восстановления до показателя по данному тесту на уровень до операции.

Динамика результатов пробы Шульте на этапах исследования.

Показатель пробы Шульте также отличался во всех группах пациентов через 1 месяц. Через месяц исследования состояние когнитивной функции у пациентов с пробы Шульте достоверно улучшилось, но полностью восстановился через месяц у пациентов 3 группы на уровень до операции и у пациентов 1 группы на уровень максимально возможного значения по этому тесту. У пациентов 1 группы через три месяца после операции показатель пробы Шульте полностью восстановился по сравнению с максимально возможным результатом по тесту и был на $8,8 \%$ ниже значений до операции у пациентов этой группы. У пациентов 3 групп через три месяца после операции показатель пробы Шульте полностью восстановился по сравнению со значениями данного теста к операции у пациентов этой группы. У пациентов 2 группы через три месяца после операции показатель пробы Шульте был на $38,2 \%$ ниже максимально возможного значения по данному тесту и на $16,9 \%$ ниже значения по данному тесту в период до операции у пациентов этой группы. У пациентов всех групп в короткие сроки наблюдений существенной разницы скорости восстановления не было. Восстановление было постепенным, с полным восстановления до уровня когнитивных нарушений по данному тесту на уровень до операции.

Выводы. По результатам динамики изменения теста по шкале MMSE, у пациентов среднего возраста наблюдалась динамика ухудшения состояния когнитивной функции в меньшей степени, чем у пациентов молодого возраста, что, возможно связано с возрастными особенностями пластичности когнитивной функции. У пациентов среднего возраста, по результатам шкалы $\mathrm{FAB}$, в данный период исследования было на одном уровне с показателями у пациентов молодого возраста, что, связано с возрастными особенностями пластичности когнитивной функции. По тесту рисования часов, существенной разницы скорости восстановления не было, оно было постепенным, но полного восстановления не наблюдалось. По тесту запоминания 10 слов и показателям пробы Шульте восстановление было постепенным, с полным восстановлением показателя на уровень до операции.

\section{ЛИТЕРАТУРА}

1. Cognitive dysfunction 1-2 years after non-cardiac surgery in the tlderly / H. Abildstrom, I.S. Rasmussen, P. Rentown // Acta Anestesiol. - 2000. - Vol. 44. - P.1246-1251.

2. Усенко Л. В., Ризк Шади Ейд, Криштафор А. А. и др. Профилактика и коррекция послеоперационных когнитивных дисфункций у больных пожилого возраста // Международный неврологический журнал. - 2008. - № 3 (19). - С. 99-110.

3. Усенко Л. В., Ризк Шади Ейд, Криштафор А. А. и др. Профилактика и коррекция послеоперационных когнитивных дисфункций у больных пожилого возраста // Международный неврологический журнал. - 2008. - № 4 (20). - С. 87-94.

4. 4.Шнайдер Н. А., Шпрах В. В., Салмина А. Б. Послеоперационная когнитивная дисфункция: профилактика, диагностика, лечение. Методическое пособие для врачей. - Красноярск: Оперативная полиграфия, 2005. - 95 с.

5. 5.Давыдова Н. С. Возможные критерии прогноза нарушений мозгового кровообращения при анестезии // Вестник Интенсивной Терапии - 2004. - №5. - С.232-234.

6. Шнайдер Н. А. Новый взгляд на проблему послеоперационной когнитивной дисфункции // Журнал острые и неотложные состояния в практике врача. - 2006. - № 5. - С . 47-49.

7. Rasmussen L. S., Jonson T., Kuipers H. M. et al. Does anesthesia caese postoperative cognitive disfunction? A randomized study of regional versus general anesthesia in 438 elderly patients // Acta Anesth. Scand. 2003. - V.47. - No.9. - P.1188-1194.

8. Шнайдер Н. А., Салмина А. Б. Неврологические осложнения общей анестезии. - Красноярск: КрасГМА, 2004. - 383 с.

9. Захаров В. В. Когнитивные расстройства в пожилом и старческом возрасте: методическое пособие для врачей/ В. В. Захаров, Н.Ню Яхно - Москва, 2005. - 71с. 